\title{
Managerial Modes of Conflict Resolution in the Banking Industry
}

\section{A D Slabbert}

Faculty of Management, Cape Technikon

\section{ABSTRACT}

The Thomas Killman Conflict Mode Instrument is widely used to assess conflict management styles. The instrument uses two parameters, i.e. assertiveness and cooperation, resulting in five distinct styles: avoiding, competing, collaborating, accommodating and compromising. Twenty five senior- and twenty five junior managers in the banking industry (2 particular companies) completed the questionnaire, answering in the context of their relationships with each other. Results indicate significant differences between the two groups. Senior management prefers the assertive styles (competing and collaborating) with scant attention to cooperation, while middle management primarily uses the avoiding style, which is both uncooperative and unassertive. The implications of these findings are discussed, leading to particular recommendations.

JEL J50. M12

\section{INTRODUCTION}

Conflict appears to be an integral component of human functioning - it has manifested in diverse formats for millennia. The term could be classified according to a myriad of parameters and variables, and this creates difficulty in formulating an operational and exact definition. Robbins (1998) believes that certain common denominators underlie most definitions, and that formal definitions should strive for broad flexibility. He accordingly develops the following definition:

"... a process that begins when one party perceives that another party has negatively affected, or is about to negatively affect, something that the first party cares about" (1998: 434).

Throughout history, particular perceptions/philosophies have developed regarding conflict. The so-called traditional view, which was primarily based on attitudes that prevailed about group behaviour in the 1930s and 1940s, maintained a rigid, formalistic approach to conflict. Conflict was regarded as 
dysfunctional and had to be avoided at all costs by roleplayers. The more modern approach originated in the discourses and precepts of the human relations theorists, who stipulated that conflict is a natural phenomenon, that it is inevitable and that it should be managed as such (Reyneke, 1997).

The paradigms of these theorists have become somewhat archaic - it is now generally accepted that conflict should be measured in terms of functionality, i.e. whether it manifests as functional and contributes to personal/organisational growth, or whether it is dysfunctional and impedes group/individual performance (Caudron, 2000; Alper et al., 2000 and Neuper, 1996).

It remains a fair assumption that if an individual experiences conflict as destructive and dysfunctional, subsequent behaviour will be adjusted correspondingly, and that such behaviour will impact negatively on the individual's wider sphere of influence (Womack, 1988). This assumption is supported by amongst others Reyneke (1997) as well as Song et al. (2000). Under such conditions, within organisational context, non-achievement of stated goals and non-performance then becomes both a feasible and logical consequence. The traditional triad of individual, group and organisational goals and objectives remains constant and in an inviolate relationship. Essentially, this means that dysfunctional conflict between individuals will impact negatively on organisational performance (Zartman, 2000 and Drolet et al,, 2000).

Reyneke (1997) stipulates that organisations generally display an inability to manage conflict constructively - such organisations would primarily be centred in the traditional mindset of conflict (see above). Conflict within the framework of such organisations would in essence be regarded as a threat, and would impact on interpersonal relations and decision-making. These could have a significant impact on the manner in which the organisation functions.

An assumption can be formulated that these two elements of organisational life, (relationships and decision-making styles), could theoretically be linked to productivity and performance. Although direct statistical relationships in this context are difficult to detect, it remains a fairly safe assumption (Neuper, 1996 and Volkema et al., 1995).

A complex array of variables determines the nature of these constructs (interpersonal relationships and decision-making styles). Variables such as personality, power, reward base, attitudes and perception operate, and eventually determine behaviour. It is not the intention of this paper to attempt even a superficial approach to understanding these variables. Rather, the premise is that ineffective or dysfunctional relationships impact negatively on 
decision-making and invariably results in impaired organisational performance (Kets-de-Vries, 1999).

The conclusion that should therefore be formulated is that dysfunctional conflict should be regarded as a contra indication for maximal performance within organisational context.

In a historical sense, certain types of organisations exhibit traditional hierarchical type behaviour. Reporting lines, authority bases and the utilisation thereof, as well as definite bureaucratic systems remain at the innermost core of such organisations. In the postmodern economic climate of instinctual and rapid change, globalisation, emerging and rapidly discarded technology and such, organisations such as these become a danger to themselves. They are archaic and run the risk of losing touch with the harsh reality of the business environment they operate in (Alper et al., 2000).

In this context, two such organisations were identified as exhibiting certain of these characteristics mentioned above. In South Africa, the banking and financial sectors possess an extended history which displays a number of the behavioural elements mentioned above. The banking sector is highly regulated and appears to function in a traditional, somewhat mechanistic fashion. Less than a decade ago, four major banks amalgamated into one central organisation, effecting the emergence of a major role player within the industry.

Although centralised control, domination of the industry, as well as the absence of a significant number of primary competitors would ensure organisational success, it can be stated that productivity and organisational performance could be enhanced further by the utilisation of sound interpersonal skills and decisionmaking techniques, as indicated by the referenced literature. In addition, as was alluded to earlier, conflict management styles are directly affected by interpersonal relationships and decision-making.

\section{RESEARCH METHODOLOGY}

\section{Measure}

To assess existing paradigms within this particular industry, an evaluative approach was designed as follow. Data was collected through the standardised Thomas Kilmann Conflict mode instrument, which is described as an untimed, self-scoring forced choice instrument designed to measure five distinct modes of conflict-management behaviour. Subjects are requested to consider situations in which they may find their needs to oppose that of another party. In 
each item the subject has to exercise a choice between two statements, reflecting a personal preference for a particular statement. An example of this is the following:

A. "I try to avoid creating unpleasantness for myself."

B. "I try to win my position."

Subjects responded to each question by choosing either an $\mathrm{A}$ or $\mathrm{B}$ reaction which would characterise their dominant response in a particular situation in relation to their superior or subordinate. It was stressed that subjects should restrict their answers to the relationship between themselves and their superiors or subordinates. It is generally accepted that conflict management styles would differ per situation/person, but for the purpose of this study, subjects had to focus exclusively on this particular relationship.

The questionnaire is widely regarded as the world's leading conflict resolution assessment tool (http://www.SkillsOne.com). It has been utilised extensively by a wide range of roleplayers, ranging from business consultants, psychologists, organisation development facilitators, through to church groups. An electronic search reveals 2390 sites dedicated to the use of the instrument.

The instrument was designed to assess an individual's behaviour in conflict situations, and provides a profile of individuals and groups that illustrates the repertoire of conflict management skills. Several construed benefits exist for groups and individuals:

- developing of an awareness with regard to preferred modes of conflict management

- developing of a realistic perspective conceming various sources of conflict and its impact on the organisation

- developing increased effectiveness in conflict management

- developing an awareness of the need for flexibility in different conflict situations (Thomas, 1992).

The five possible styles which are measured by the questionnaire are defined in a matrix format, and determined by relative scores on two axes, i.e. assertiveness and cooperation. The competing style is assertive and uncooperative and could be regarded as utilising personal or positional power. Accommodating is regarded as unassertive and cooperative, a style wherein the individual allows the other party to dominate. Avoiding is both unassertive and uncooperative, and is normally characterised by the individual actively avoiding any type of action. Collaborating is assertive and cooperative and represents direct attempts at conflict resolution. Compromising is intermediate in both 
assertiveness and cooperation, and is designed to partially satisfy the needs of both parties.

The configuration of the styles is depicted in the figure below:

\section{Figure 1 Conflict mode instrument}

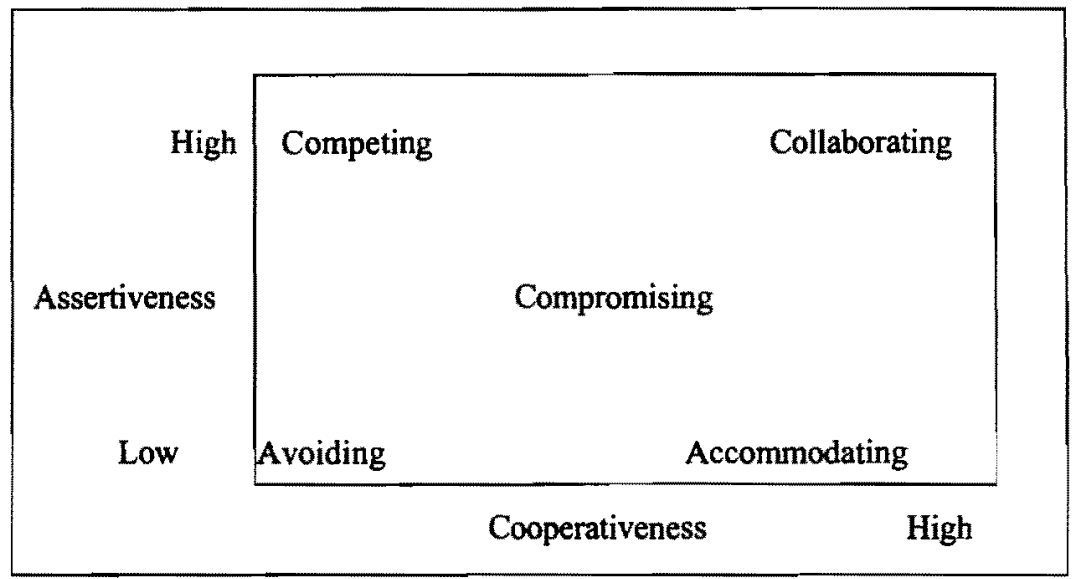

Source: Thomas, 1992

The primary intention of the instrument is to identify an individual's dominant style of conflict management. However, it is also of paramount importance for individuals or groups to acquire style flexibility or variation in responding to various situations. In this sense, different situations would require different modes of conflict resolution, for instance:

- competing could be used when quick, decisive action is vital.

- accommodating could be utilised in order to preserve harmony and to avoid disnuption

- avoiding is relevant when an issue is trivial or only of passing importance

- collaboration is used in order to find integrative solutions when the concerns of both parties are too important to be compromised

- compromising is used in order to achieve temporary settlement of complex issues (Thomas, 1992).

Style variability and flexibility therefore becomes an important outcome of exposure to the questionnaire. 


\section{Subjects}

Subjects were drawn from staff members at two large national banks. The decision to include at least two distinct organisations was based on the premise that results could conceivably be skewed through prevailing organisational cultures. It is improbable that two independent organisations, though operating in the same industry, would both present with such a contaminating variable of this nature.

Subjects were randomly drawn from two specific organisational groupings, i.e. senior management and middle management. The sample was drawn from particular branches of the organisations, resulting in a fixed definition of the subject groups, in order to circumvent contaminating variables in the form of disparate organisational levels. All senior managers in the subject sample had the job title: branch manager; whereas middle management was classified as assistant branch managers. The nomenclature senior/middle management is used in this article and should be considered in branch context. The resultant sample therefore consisted of individuals from only two job gradings. The final sample comprised 50 subjects, evenly distributed between senior and middle managers, i.e. 25 subjects per sample group.

The researcher took a decision not to effect any further control with regard to further variables which could impact on the results. The research design simply focussed on the inclusion of the two subject groupings, without controlling for biographical variables such as gender, age, length of tenure or other nuisance variables. For illustrative purposes only, nineteen (19) of the 25 senior managers were male, with a corresponding figure of 13 applying to the middle manager sample. The average length of tenure for the senior managers was 12.3 years, and 8.7 years for the middle managers.

\section{RESULTS}

\section{Data analysis}

A correlational analysis within and between groups was conducted, in order to determine:

a) predominant styles within groups; and b) fundamental style differences between groups. A two factor analysis of variance with replication was utilised. The data displayed significant differences, all at the 1 per cent level of significance. 
For illustrative purposes, the average scores obtained by subjects are displayed in Table 1 below:

Table 1 illustrates significant differences between the two groups. A cursory inspection indicates that the utilisation of styles if mutually exclusive.

Senior management subjects cluster around the assertive modes, i.e. competing and collaborating, while these styles enjoy a very low priority amongst middle managers. Conversely, the latter group of subjects exhibit a preference for the unassertive modes, i.e. avoiding and accommodation, whereas senior managers rarely utilise these styles. The conclusion here is that senior managers are significantly more assertive in their interaction with their subordinates than vice versa.

Table 2 illustrates the differences within groups (middle management), and is based on a single factor analysis of variance:

Table 2 Middle managers - style variation

\begin{tabular}{|l|r|r|r|r|r|r|}
\hline $\begin{array}{c}\text { Source of } \\
\text { variation }\end{array}$ & $\begin{array}{c}\text { Sum of } \\
\text { squares }\end{array}$ & $\begin{array}{c}\text { Degrees of } \\
\text { freedom }\end{array}$ & $\begin{array}{c}\text { Mean } \\
\text { Square }\end{array}$ & F & P-Value & F-Crit \\
\hline Between groups & 646.27 & 4 & 161.57 & 39.19 & $5.87 \mathrm{E}-21$ & 3.4795 \\
\hline Within groups & 494.72 & 120 & 4.12 & & & \\
\hline Total & 1140.99 & 124 & & & & \\
\hline
\end{tabular}

An analysis of the above indicates a strong degree of homogeneity between individual subjects, although variation is noticeable. However, specific and clear differences emerge between mode utilisation, and the p-value of 5.87E-21 indicates significant differences in terms of mode preferences.

Table 3 illustrates the differences within groups (Senior management) and is based on a single factor analysis of variance:

A similar pattem to that of middle managers emerged - homogeneity between individual subjects, while the p-value of 9.25E-25 stipulates clear differences in terms of the utilisation of modes. 
Table 3 Senior managers - style variation

\begin{tabular}{|l|r|r|r|r|r|r|}
\hline \multicolumn{1}{|c|}{$\begin{array}{c}\text { Source of } \\
\text { variation }\end{array}$} & $\begin{array}{c}\text { Sum of } \\
\text { squares }\end{array}$ & $\begin{array}{c}\text { Degrees } \\
\text { of } \\
\text { freedom }\end{array}$ & $\begin{array}{c}\text { Mean } \\
\text { Square }\end{array}$ & F & P-Value & F-Crit \\
\hline Between groups & 876.24 & 4 & 219.06 & 50.19 & $9.25 \mathrm{E}-21$ & 3.4795 \\
\hline Within groups & 523.76 & 120 & 4,36 & & & \\
\hline Total & 1400 & 124 & & & & \\
\hline
\end{tabular}

Table 4 addresses the crucial issue of the comparative style utilisation of the two subject groups:

Table 4 Senior vs. middle managers - style utilisation

\begin{tabular}{|l|c|c|c|c|c|c|}
\hline $\begin{array}{l}\text { Source of } \\
\text { variation }\end{array}$ & $\begin{array}{c}\text { Sum of } \\
\text { squares }\end{array}$ & $\begin{array}{c}\text { Degrees } \\
\text { of } \\
\text { freedom }\end{array}$ & $\begin{array}{c}\text { Mean } \\
\text { Square }\end{array}$ & F & P-Value & F-Crit \\
\hline Rows & 52.889 & 24 & 2.204 & 0.534 & 0.96427 & 1.88967 \\
\hline Columns & 1031.04 & 8 & 128.88 & 31.241 & $5.09 \mathrm{E}-31$ & 2.60505 \\
\hline
\end{tabular}

Definitive differences between the two groups appear, as indicated by the Pvalue of 5.09E-31. Again, no fundamental variation exist within the groups, which leads to the conclusion that a fairly uniform style of mode utilisation exists. It is clear however, that the two groups of subjects exhibit marked differences in mode preferences, a finding which is supported by a cursory examination of Table 1 (simple averages).

\section{DISCUSSION}

The results which were obtained from the data sample strongly support initial expectations, i.e. that there would be significant differences in the modes of conflict resolution on different organisational levels within traditional organisations. It is apparent that the styles of conflict resolution relate to the existence of a strict organisational hierarchy, with very little deviation within both organisational groupings, i.e. senior and middle managers. In summative form: senior managers utilise high assertiveness/low cooperation styles, while middle managers (their subordinates within the parameters of the present study) manifest directly opposite styles, i.e. low assertiveness/high.cooperation. 
This scenario illustrates a system which appears to be distinctly archaic within the context of post modem business management. A distinctly autocratic management style appears to exist, with approval and/or covert condolence from both parties within the relationship. The culture which evolves from this type of relationship essentially portrays a downward cascading of orders or instructions and silent acquiescence from the more junior employees. Within these relationships, there would be little opportunity for initiative, self direction and autonomy - a situation which is in stark contrast with the motivational factors as identified by Herzberg decades earlier.

The relationship which appears to emerge is one of dominance - subservience, and in all probability enjoys tacit approval from role players. The rationale behind this statement relates to the extreme uniformity that emerged from the responses of the subjects, as was evidenced by Tables 2 and 3. It is improbable that such extreme uniformity would materialise in a boundary free environment, due to the influence that personality and other variables would effect in such a milieu. Instead, a more profound and distinct heterogeneity in conflict management styles would be at the order of the day. The extent and degree of the uniformity of responses would therefore indicate that another variable operates within this particular environment: a somewhat sinister variable which impacts very deeply not only on individual personalities, but also on organisational culture and climate. This variable (acquiescence to authority) creates a boundary driven organisation, and limits the behavioural scope of employees.

The nature of the response set illustrates a further reality which deserves pertinent attention: if these particular behaviours manifest in such a defined manner as it did in this case, it serves to illustrate that there is a permanency attached to them. This reality points to the fact that behaviour, once acquired, seems to perpetuate itself in a predictive cyclical fashion, i.e. middle managers obey without question until they are promoted (which they probably will, due to the fact that they do not disturb the organisational serenity). At that stage, organisational culture, as well as a probable sense of entitlement, empowers them to adopt a more assertive/less cooperative behavioural style.

A host of models/techniques exist which can be applied to human behaviour within organisational context. Taylor (2001) provides interesting viewpoints on the amalgamation of Thomas-Kilmann and the Four Functions model of Jung. In essence, Jung postulates four different levels of perception or behaviour, namely thinking, sensing, intuition and feeling. Taylor (2001) correlates these respectively with competing; compromising; avoiding, while accommodation and collaboration both manifests on the feeling level. 
Within this framework, certain perspectives emerge: integrated human functioning is a result of an interplay between the four levels. If subjects in this particular sample precludes certain of these levels in terms of their interaction with each other, an assumption can clearly be made that the nature of the dyadic relationships is impoverished in nature. Impoverished relationships per definition are not building blocks for a healthy organisational culture and could conceivably impact on organisational performance.

In addition, it appears as if senior managers operate primarily on the thinking level, while middle managers operate on the intuitive level. This indicates that senior managers consciously construct a set of behaviours, while middle managers operate on a more subliminal (anxiety driven?) level. Again, contra indications for organisational success are evident in this scenario.

Both organisations that participated in the study, have an active and dynamic human resource function, with a strong focus on management development and training. Despite the functioning of these departments and their inputs, subjects still displayed responses to the questionnaire which are at complete variance with the philosophies which Human Resources attempt to engender in employees. This raises two important questions: 1) do the results of this study indicate that the Human Resource function in totality is being indicted as ineffective and irrelevant? or 2) do the results point to the possibility that the Human Resource function at these institutions has become a victim of the authority/subservience paradigm? It is not conceivable that the first question deserves serious attentional focus, and the conclusion should therefore be reached that the latter is true, and has become a most unfortunate reality at these institutions. Although extrapolation to other institutions within the same industry cannot be attempted, certain puzzling questions become relevant. A Human Resource function's primary role, if it desires to be a functional strategic partner in its organisation, is to identify and rectify dysfunctional behaviours (Boase, 1997), and not to become a victim of such processes.

In an economic sense, it is apparent that these organisations are not functioning at an optimal level. Several authors have indicated an inverse relationship between dysfunctional conflict management and organisational performance (Caudron, 2000; Alper et al., 2000; Song et al., 2000 and Reyneke, 1997). Despite impressive financial year-end reports to shareholders, it is apparent that organisational climate within these organisations yields a less-than-satisfactory return.

It would not be advisable to use the present study as a generic base for generalisation, as the subject sample is relatively small. However, it is pertinent to again refer to the homogeneity of the responses: it is conceivable that this 
could be a reflection of the wider status quo within the banking industry. It is therefore imperative to undertake broad based research within this industry in order to determine the actual state of affairs. This industry is a major roleplayer within the South African economy, and its functioning has important implications for a wide array of associated organisations within the economic framework.

South Africa is not in a position to economically dissociate itself from other international role players. Within the confined limits of a constrained economy and semi-monopolism of the industry, the two organisations in the present study may continue to declare impressive financial returns. Globally however, archaic management styles and boundary restrictive organisational cultures will contribute to unsatisfactory, and indeed unnoticed, international performance.

It is imperative that all South African organisations realise and accept their integral responsibility towards all stakeholders. Organisational cultures which are in a sense derived from conflict resolution styles should contribute to the formation of a strong economy which cannot be denied international attention. Dysfunctional conflict resolution serves as an economic and moral indictment of all role players, and should not be tolerated.

\section{CONCLUSION}

From the preceding, it is apparent that managers at different organisational levels utilise strongly divergent modes of dealing with conflict. In addition, the conclusion could be reached that the nature of the relationships which are defined by these modes of conflict resolution, is based on authority and subservience. Finally, it emerges that managers at both levels are somewhat dogmatic and exhibit strong adherence to particular styles - there is little evidence of style variability and adaptation. These findings do not auger well for conflict resolution within these organisations, and raises a number of questions concerning issues such as organisational culture, effectiveness and eventually, productivity.

\section{REFERENCES}

1 ALPER, S., TJOSVOLD, D. \& LAW, K.S. (2000) "Conflict Management, Efficacy and Performance in Organizational Teams", Personnel Psychology, 53(3): 625-42. 
2 BELL, M.L. \& FORDE, D.R. (1999) "A Factorial Survey of Interpersonal Conflict Resolution", The Journal of Social Psychology, 139(3): 369-77.

3 BOASE, N. (1997) "HR Fundamentals: Resolving Conflict in the Workplace", People Dynamics, 15(7): 48.

4 CAUDRON, S. (2000) "Keeping Team Conflict Alive: Conflict Can be a Good Thing. Here's What You Can Do to Make the Most of this Creative Force", Public Management, 82(2): 5-9.

5 CRI PSYCHOLOGISTS, Available: http://www.SkillsOne.com

6 DOMBAI, C. \& VERWEY, S. (1999) "The Impact of Organisational Culture as a Context of Interpersonal Meaning on the Management of Organisational Diversity", Communicare, 18(2): 104-31.

7 DROLET, A.L. \& MORRIS, M.W. (2000) "Rapport in Conflict Resolution: Accounting for How Face-to-Face Contact Fosters Mutual Cooperation in Mixed-Motive Conflicts", Journal of Experimental Social Psychology 36(1): 26-50.

8 FISKE, E.P. (2000) "Values and Interests in Conflict Resolution", Rural Sociology, 65(2): 331-7.

9 Jude, B. (1998) "Conflict Management: Know-How" Career Success, 11(3): 6-7.

10 KETS, D. \& MANFRED, F.R. (1999) "What's Playing in the Organizational Theater? Collusive Relationships in Management", Human Relations, 52(6): 745-73.

11 LIDEN, R. \& GRAEN, G. (1980) "Generalizability of the Vertical Dyad Linkage Model of Leadership", Academy of Management Joumal: 45165.

12 NEUPER, T.P. (1996) "Sources of Power and Conflict Handling Styles of Supervisors".

13 NICOTERA, A.M. (1993) "Beyond Two Dimensions: A Grounded Theory Model of Conflict-Handling Behavior": Management Communication Quarterly, 6(3): 282-306.

14 OLSON-BUCHANAN, J.B., DRASGOW, F. \& MOBERG, P.J. (1998) "Interactive Video Assessment of Conflict Resolution Skills", Personnel Psychology, 51(1): 1-24.

15 PHILLIPS, A.S. \& BEDEIAN, A.C. (1994) "Leader-Follower Exchange Quality: The Role of Personal and Interpersonal Attributes", Academy of Management Journal: 990-1001.

16 REYNECKE, L. (1997) "The Relation between Personality Dimensions and Styles to Handle Interpersonal Conflict".

17 ROBBINS, S.P. (1998) Organizational Behavior, Simon \& Schuster, New Jersey. 
18 SONG, X.M., XIE, J. \& DYER, B. (2000) "Antecedents and Consequences of Marketing Managers' Conflict-Handling Behaviors, Journal of Marketing, 64(1): 50-66.

19 TAYLOR, A. (2001) Models for Management, Available: http://www.arlenetaylor.org/models.htm.

20 THOMAS, K.W. (1992) "Conflict and Negotiation Processes in Organizations", In MD Dunnette \& LM Hough (eds.) Handbook of Industrial and Organizational Psychology: 651-717, Palo Alto, CA: Consulting Psychologists Press.

21 VOLKEMA, R.J. \& BERGMAN, T.J. (1995) "Conflict Styles as Indicators of Behavioral Pattems in Interpersonal Conflicts", Journal of Social Psychology, 135(1): 5, 11, 2 charts.

22 VROOM, V.H. \& JAGO, A.G. (1995) "Situation Effects and Levels of Analysis in the Study of Leader Participation", Leadership Quarterly: 169-81.

23 WOMACK, D.F. (1998) "Assessing the Thomas-Kilman Conflict MODE Survey", Management Communication Quarterly, 1(3): 321-49.

24 ZARTMAN, L.W. (2000) "Conflict Management: The Long and the Short of It", SAIS Review, 20(1): 227-35. 